Sharif University of Technology
Scientia Iranica
SCIENTIA $\quad \begin{gathered}\text { Transactions D: Computer Science Es Engineering and Electrical Engineering } \\ \text { I RAN I C A }\end{gathered}$

Invited Paper

\title{
Reliability enhancement of active distribution grids via emergency V2G programs: An analytical cost/worth evaluation framework
}

\author{
H. Farzin* and M. Monadi \\ Department of Electrical Engineering, Faculty of Engineering, Shahid Chamran University of Ahvaz, Ahvaz, P.O. Box 61357-43337,
} Iran.

Received 2 August 2019; accepted 17 September 2019

\author{
KEYWORDS \\ Active distribution \\ system; \\ Analytical method; \\ Cost/worth analysis; \\ Reliability; \\ Vehicle-to-Grid \\ (V2G).
}

\begin{abstract}
In this paper, an analytical cost/worth evaluation framework is presented for determination of optimal reliability enhancement plan in active distribution systems. In this regard, it is assumed that emergency Vehicle-to-Grid (V2G) programs provided by parking lots will be deployed for supplying loads during network outages and the optimal number of participating Electric Vehicles (EVs) is determined using a reliability cost/worth method. Through the presented framework, annual social costs of the distribution system, including investment costs, operating costs, revenues, and Customer Interruption Costs (CIC), are minimized. To this aim, an estimation procedure of different cost terms will be introduced. Moreover, an appropriate analytical framework is presented for the calculation of CIC and a simple method is developed for estimating the financial costs of emergency V2G programs. The presented framework is implemented for a test system, various emergency V2G programs are investigated, and the results are discussed.

(C) 2019 Sharif University of Technology. All rights reserved.
\end{abstract}

\section{Introduction}

Electric Vehicles (EVs) are expected to constitute a substantial portion of transportation sector in the upcoming years. As the number of EVs rises, a new major load will be introduced to power system [1]. On the other hand, they can assist the grid by the Vehicleto-Grid (V2G) capability [2]. V2G-capable EVs may be effectively involved in network operation as dispersed energy storage systems and they can play the role of backup units for Renewable Energy Sources (RESs).

The V2G capability of EVs can provide new op-

*. Corresponding author. Tel.: +986133330011 E-mail addresses: Farzin@scu.ac.ir (H. Farzin); M.Monadi@scu.ac.ir (M. Monadi)

doi: 10.24200 /sci. 2019.54158 .3624 portunities for power system operators in confrontation with issues such as reactive power support, active power regulation, and peak load shaving [3-6].

Generally, EV users can charge their vehicles at home or public parking lots [7]. At homes, EVs are usually charged with low charging currents. By contrast, at public parking decks, they can be charged more rapidly via fast chargers [8]. Moreover, these entities can effectively play the role of EV fleet aggregators [9]. This leads to significant savings in the required investment in electrical and data communication infrastructures and facilitate the coordinated management of EVs. Therefore, public parking lots have been considered as proper candidates for providing the required charging/discharging services to EVs [10].

Since there are usually a large number of EVs available in a parking lot at any time, a significant amount of power can be injected from the parked EV 
batteries into the grid during emergency conditions. This leads to a substantial improvement in the reliability level of distribution systems, especially in the presence of RESs.

Despite numerous technical and financial studies on the role of $\mathrm{V} 2 \mathrm{G}$ programs in modern power systems, the research focusing on reliability impacts of aggregated EVs fleet is limited. In this context, Lin et al. [11] and Farzin et al. [12] proposed a simulation-based approach to assessing the reliability impacts of V2Gcapable EVs in Battery Exchange (BE) mode. Also, some adequacy studies of generation and distribution systems in the presence of EVs fleet have been carried out [13-15]. Moreover, the role of public parking lots in reliability improvement of active distribution systems has been investigated [7]. Reliability improvement of renewable-based energy hubs in the presence of emergency V2G programs has been investigated via an analytical approach [16].

All the above-mentioned studies have demonstrated the significant potential of emergency V2G programs for reliability enhancement of power grid. However, since battery accounts for a remarkable portion of the capital cost of EVs, concerns about increased battery wear due to more frequent charging/discharging cycles has remained as a major obstacle to widespread implementation of such programs $[17,18]$.

Before implementing the emergency $\mathrm{V} 2 \mathrm{G}$ programs, all the associated costs/benefits should be accurately modeled and analyzed. Accordingly, a cost/worth analysis framework will be presented in this paper. This framework can be used as a practical tool for assessing the viability of emergency V2G programs for reliability enhancement of active distribution systems from a financial point of view.

Incurred costs by implementation of the emergency V2G program include investment and operating costs minus the obtained revenues. On the other hand, implementation of such programs results in reliability worth, which is reflected in reduced Customer Interruption Costs (CIC). Therefore, procedures are introduced for estimation of different cost terms. Moreover, an appropriate analytical method is presented for the calculation of CIC. This method addresses different probabilistic factors that affect the interruption cost of customers, while trying to reduce the associated computational burden. It estimates the available energy of batteries considering random behavior of $\mathrm{EV}$ users as well as their charging/discharging patterns. Moreover, variability of demand and output power of Distributed Generation (DG) units is considered in the estimation of the incurred interruption costs. It should be noted that identification of the optimal V2G program through reliability cost/worth evaluation imposes a high computational burden and the analytical nature of the proposed CIC estimation framework facilitates its application. Moreover, note that the introduced analytical framework is intended for rough analysis and comparison of different $\mathrm{V} 2 \mathrm{G}$ programs, and the final decision can be made using more accurate methods such as Monte Carlo simulation [7,19].

As mentioned earlier, operating costs of the introduced programs are mainly attributed to the wear cost of $\mathrm{EV}$ batteries due to discharge actions during emergency events. In this context, a simple model based on a previous study carried out by the authors [18] is introduced and the associated costs are estimated considering battery specifications, EV driving behaviors, charge scheduling strategies, and the usage patterns.

Once all cost/revenue terms have been evaluated, they are annualized and the optimal emergency $\mathrm{V} 2 \mathrm{G}$ program is determined based on the annualized cost/worth comparison. Note that although the proposed framework in this paper is designed for general purposes, the focus is on implementation of the emergency V2G programs in a distribution system containing multiple microgrids, i.e., multi-microgrid (MMG) systems [20,21]. However, as will be explained, this framework can be readily applied to any distribution grid.

In summary, the major contributions of this paper can be listed as follows:

- A framework based on reliability cost/worth analysis is presented for determining the optimal emergency V2G program in a distribution system;

- An appropriate analytical approach is introduced to the estimation of CIC. In this framework, random behavior of EV users as well as their charging/discharging patterns are taken into account. Moreover, operating modes of different microgrids (MGs)/sections are identified and their impacts on CIC are considered;

- The costs associated with EV batteries wear due to more frequent charging/discharging in emergency V2G programs are estimated using practical assumptions;

- Extensive case studies are provided to demonstrate the applicability of the proposed framework and to identify the main factors affecting the viability of adopting emergency $\mathrm{V} 2 \mathrm{G}$ programs for reliability reinforcement.

The remainder of this paper is organized as follows. Section 2 introduces the reliability cost/worth method and presents the formulation. Sections 3 and 4 introduce the proposed analytical framework for CIC estimation and battery wear costs, respectively. The presented framework for cost/worth analysis is described in Section 5. Finally, case studies are provided in Section 6, followed by conclusions in Section 7 . 


\section{Outline of the proposed framework}

\subsection{Reliability cost/worth method}

Reliability cost/worth analysis is an effective tool for making optimal decisions about design, operation, and performance of power systems. In this paper, it is assumed that Distribution System Operator (DSO) intends to improve reliability of the active distribution system via implementation of emergency V2G programs. Before deciding about the optimal system reinforcement plan, different incurred costs as well as the expected benefits for customers should be carefully analyzed and evaluated [22]. Reliability enhancement costs can be broadly classified as DSO costs and CIC. DSO costs can be categorized as investment and operation costs. On the other hand, CIC mainly depends on customer type and outage durations. These costs are usually evaluated using Sector Customer Damage Function (SCDF) or Combined Customer Damage Function (CCDF) [23]. These characteristic curves, which provide interruption costs as a function of outage duration, can be used for estimation of CIC in the system.

Implementing reliability enhancement plans in distribution systems usually increases DSO costs and, on the other hand, reduces CIC. This concept is schematically illustrated in Figure 1. The figure depicts the general variation pattern of different cost terms as a function of reliability level. In reliability cost/worth method, the optimal level of reliability is determined such that social costs of the system are minimized [22]. Such a level is specified in Figure 1 using a dotted line. As can be observed, if this reliability level is maintained, social cost of distribution system, which is composed of DSO and CIC, is at its minimum value.

\subsection{Model formulation}

Based on the previous discussions, optimal emergency V2G program can be determined using the following optimization problem:

$$
\operatorname{Min} S C=I C+O C+C I C-R e v \text {, }
$$

where SC and OC denote annual Social Cost and

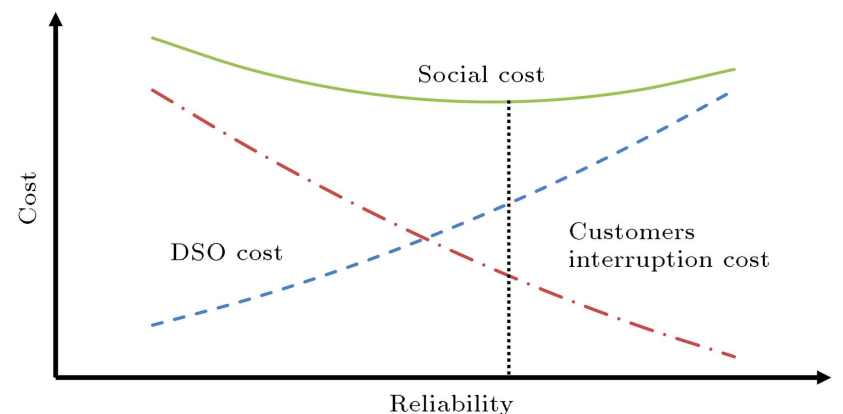

Figure 1. Schematic illustration of cost variation pattern versus reliability [24]. annual Operating Cost of the distribution system, respectively. In addition, IC and Rev respectively represent annualized Investment Cost required for implementation of emergency V2G programs and annual Revenue that DSO obtains by their implementation. It should be noted that in this paper, we intend to determine the optimal number of EVs that should participate in the emergency V2G programs. In order to determine the optimal number of EVs, Social Cost (SC) should be assessed and compared for different values of the participating EV numbers.

To this aim, it is assumed that the parking lot facility and the control and communication infrastructures required for implementation of the V2G programs are already available in the distribution grid under study. Based on this assumption, it can be confirmed that implementation of the emergency $\mathrm{V} 2 \mathrm{G}$ programs does not require new investments in the system. On the other hand, implementation of these programs does not result in revenues for the DSO. Hence, both IC and Rev will be equal to zero in this case and only the values of $\mathrm{OC}$ and CIC should be evaluated for different numbers of participating EVs.

CIC can be approximated using the following equation [22]:

$$
C I C=E N S \times V O L L,
$$

where ENS is the index of Expected Non-Served Energy in the distribution system and VOLL is the Value Of Lost Load for the combined loads of the distribution system. The value of VOLL can be obtained for each system considering the composition of different load types as well as the associated SCDF curves [22]. Once the value of VOLL (also known as IEAR [23]) has been calculated for a specific system, the expected value of CIC can be easily estimated using Eq. (2) without introducing great inaccuracies [22]. It is noteworthy that CIC estimation can be made as accurate as needed using detailed Monte Carlo simulation of the system [23].

The approximate analytical method for estimation of ENS will be introduced in the following section. Moreover, a simple procedure is presented in Section 4 for estimating annual operating cost associated with the implementation of the emergency V2G programs.

\section{Proposed analytical method for CIC estimation}

3.1. Available energy modeling of a parking lot In order to obtain the expected value of available energy of EVs in a parking lot, in the first step, the charging behavior of EVs during normal operation of the grid should be modeled. Charge scheduling of a V2G-capable EV can be carried out using the model 
Table 1. Parking scenarios.

\begin{tabular}{ccccccccccc}
\hline Charging start time & 14 & 23 & 22 & 21 & 20 & 19 & 18 & 17 & 16 & 15 \\
Parking duration & 21 & 10 & 11 & 12 & 13 & 14 & 15 & 15 & 17 & 20 \\
Probability of the scenario & 0.049 & 0.043 & 0.079 & 0.109 & 0.120 & 0.146 & 0.164 & 0.120 & 0.119 & 0.051 \\
\hline
\end{tabular}

Table 2. Probabilities of different battery SOCs at charging start time.

\begin{tabular}{llllll} 
Initial SOC & 0.1 & 0.2 & 0.3 & 0.4 & 0.5 \\
Probability & 0.2 & 0.3 & 0.3 & 0.2 & 0.1 \\
\hline
\end{tabular}

presented in the previous work of the authors [7]. It is a Mixed-Integer Linear Programming (MILP) model, in which total electricity cost for battery charging minus the power discharge reward based on feed-intariff is minimized over the scheduling interval [13]. The constraints include maximum and minimum allowable charge/discharge power as well as maximum and minimum allowable State Of Charge (SOC) levels. As discussed by the authors previously [7], this formulation is for general purposes and can be readily modified to model different charge scheduling schemes. In this paper, 4 different charge scheduling schemes are considered as follows:

- No V2G, in which total charging costs are minimized considering the electricity tariff. In this case, V2G capability is not used;

- $V 2 G$, by which charging is controlled according to the above explanations;

- Uniform Charging, where total required charging energy of the EV is uniformly received over the entire parking period. In other words, charging power is constant over the whole parking period;

- Dumb Charging, in which the EV is charged with maximum allowable charging power until it reaches the desired SOC level. As a note, the desired SOC level in this paper is assumed to be $100 \%$.

A major factor affecting the available energy of the parking lot is the random driving behavior of $\mathrm{EV}$ users. The pertinent data associated with charging periods of EVs in parking lots can be collected through surveys. In this paper, these data are extracted from the National Household Travel Survey (NHTS) [25]. The database includes starting and ending times of daily travels, which can be respectively regarded as the ending and starting times of charging periods at the parking lot [7]. Ten parking scenarios extracted from NHTS that have the highest probabilities are used for the modeling purpose in this paper. These parking scenarios are summarized in Table 1. Moreover, the scenarios associated with SOC level of EVs at the charging start time are adopted from the previous study [7] and summarized in Table 2 .

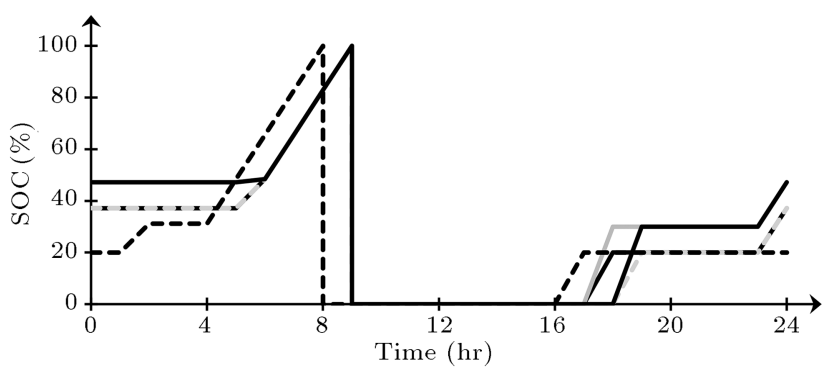

Figure 2. Five hourly energy profile scenarios with the highest probabilities (case of "No V2G").

In the next step, the profiles of EV available energy associated with different charging scenarios are obtained by solving the introduced charge scheduling problem. Assuming that initial SOCs and driving patterns are independent from each other, the probability of each available EV energy profile can be calculated by multiplying the associated probabilities of initial SOC and parking scenarios. Considering ten parking scenarios and five initial SOC scenarios, a total number of $50 \mathrm{EV}$ energy profile scenarios will be generated. As an example for the case of "No V2G," 5 hourly energy profile scenarios with the highest probabilities are depicted in Figure 2.

Subsequently, EV energy profile scenarios are weighted by their probabilities and summed up to yield the expected values of EV energy profiles as follows:

$$
E A E_{v}(t)=\sum_{k} p_{k} E_{k, v}(t)
$$

where $E A E_{v}(t)$ denotes the expected value of the available EV energy at time $t, v$ denotes EV type, $k$ is the index of energy profile scenarios, and $E_{k, v}$ is the available EV energy in scenario $k$. Expected values of the available EV energy for different charge scheduling schemes have been obtained using the introduced method and the obtained results are illustrated in Figure 3. EV battery and charging specifications are adopted from the previous study [18].

Finally, the number of participating EVs should be multiplied by the expected value of available energy profile for a single EV to estimate total available energy of the parking lot as follows:

$$
T A E(t)=\sum_{v} N_{v} E A E_{v}(t),
$$

where $T A E(t)$ denotes the expected value of total available energy of the parking lot and $N_{v}$ is the 


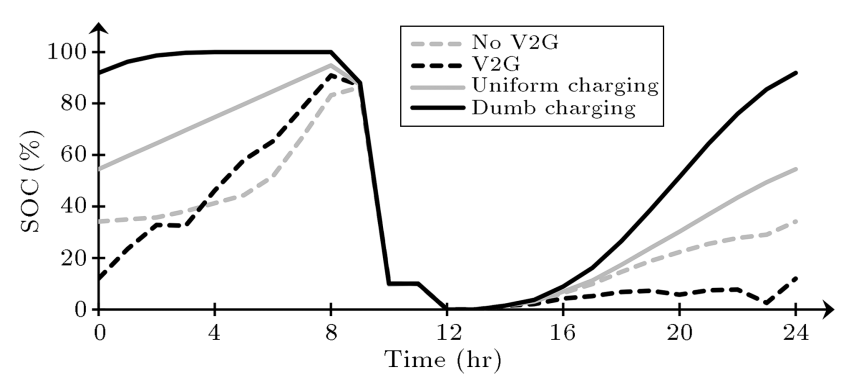

Figure 3. Expected energy profile in different charging schemes.

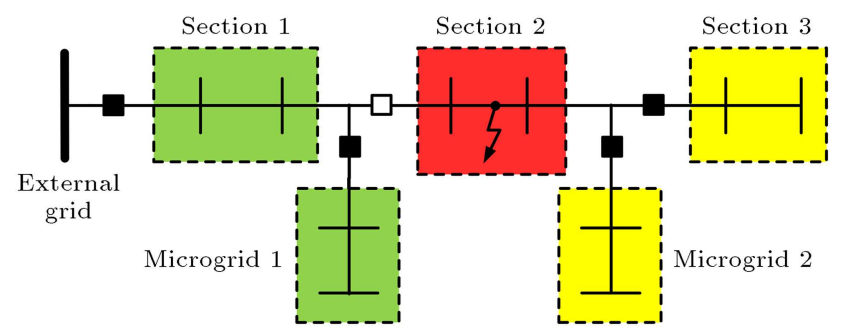

Figure 4. Sample system for illustrating MG/section operating modes [24].

number of EVs of type $v$ that participate in emergency $\mathrm{V} 2 \mathrm{G}$ program. In case of unavailability of specific data, EV numbers can be estimated based on the market shares of different EV types in the region [26,27]. Note that in this paper, it is assumed that all EVs are of the same type and the specifications are based on the EV considered in the previous study [18].

\subsection{Proposed analytical reliability assessment framework}

In the proposed method, the following assumptions are considered:

- Once a network component (line, busbar, etc.) fails, there will be no further failures during its repair/replacement period. This is a common assumption in analytical reliability assessment methods $[16,22]$ and can be justified by the low occurrence probability of simultaneous network components;

- The time interval between sequential network failures is long enough to neglect the impact of a failure on the subsequent one. In other words, different failures are assumed to be independent from each other. This assumption greatly simplifies the analysis since chronological simulation of energy storage systems and parking lot EVs during normal operation will not be necessary.

In the proposed framework, the active distribution system can be divided into multiple MGs/sections based on protective devices configuration $[19,26]$. A sample distribution system is shown in Figure 4, which is divided into 3 sections and 2 MGs. For estimation of ENS index, different failures of the system are analyzed based on Failure Modes and Effects Analysis (FMEA) method [22]. To this end, operating mode of each $\mathrm{MG} /$ section should be determined for each contingency and the amount of energy curtailment in the whole system should be calculated. Different MG/section operating modes are as follows:

- Grid-Connected: In this situation, the MG/section can directly or indirectly get access to the external grid after fault isolation. As a result, it can exchange power with the external grid. In this paper, it is assumed that external grid is able to fulfil the entire distribution system load and absorb the excess generated power [19,28]. Therefore, the customers within these MGs/sections will not be interrupted. Referring to Figure 4, after fault isolation in Section 2, Section 1 and MG 1 are operated in grid-connected mode;

- Islanded: In this situation, the MG/section will not be able to access the external grid after fault isolation. In this case, multiple islanded MGs/sections may form a larger island and share their available generations and storage capacities based on the agreed Outage Management Scheme (OMS) [20]. In Figure 4, both Section 3 and MG 2 are in islanded mode and can form a larger island;

- Faulted: In this situation, the MG/section has to disconnect all its loads (like Section 2 in Figure 4).

The analytical method involves analysis of different network failures and aggregation of the curtailed energy in different states of the Distributed Energy Resources (DERs). The pseudocode of the proposed algorithm is shown in Box I.

Based on this algorithm, different contingency events of the network are analyzed considering different occurrence times (different hours of the day in different months) and different availability states of DERs. In this algorithm, $m, h, s$, and $j$ are, respectively, the indices of month, hour of the day, availability state of DERs, and network component failures. Moreover, $N$ denotes the total number of states for each index, $p$ shows the associated probability, and $\lambda_{j}$ is the failure rate of network contingency event $j$. In this paper, 12 sample months and 24 sample hours are considered for numerical analysis. Therefore, the associated probabilities are equal to $1 / 12$ and $1 / 24$, respectively. Moreover, Forced Outage Rate (FOR) of each DER is considered as its outage probability [16] and simultaneous outage of DERs is neglected in this paper due to low probability. Note that DER outages with higher orders can be readily incorporated in the algorithm.

In order to calculate the value of total energy curtailment during failure of network component $j$ 


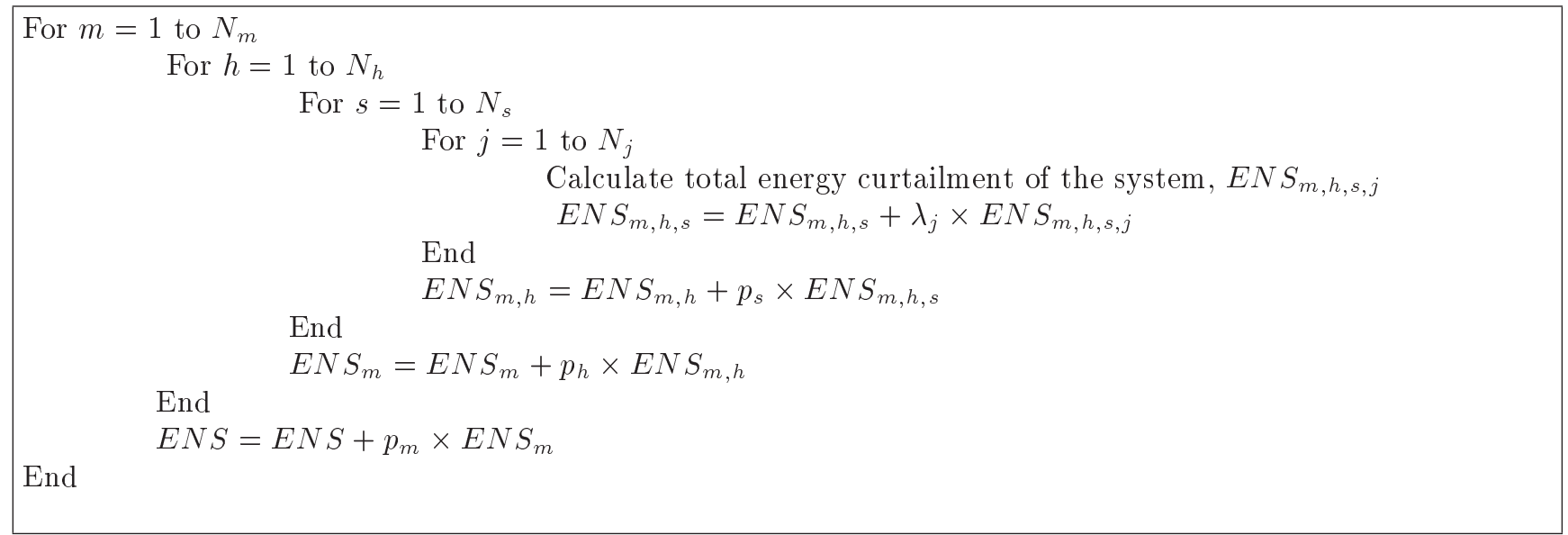

occurring at hour $h$ in month $m$ with DER outage state $s$, i.e., $E N S_{m, h, s, j}$, the following steps should be taken:

1. Operating status of different MGs/sections during the contingency should be determined;

2. As previously discussed, for grid connected sections, ENS will be equal to zero. For faulted sections, ENS will be equal to the total energy requirement during the contingency. This duration will be normally equal to the Mean Time To Repair (MTTR) of that network component [22]. As for the islanded sections, the adopted outage management scheme is simulated and total energy curtailment is recorded. For the simulations in this paper, 2 sample operating strategies are adopted from the previous study [19]. Grid-connected sections/MGs are operated in such a way that their operating costs are minimized. This is done for determination of ESS SOCs within the distribution system. On the other hand, a decentralized outage OMS is used for scheduling of islanded portions, where load curtailment costs are minimized. As for the participation of parking lot in energy management of the islanded portions, it is assumed that the available EVs energy has the lowest priority for supplying the loads. In other words, the available energy will be used only if after implementation of the OMS, there is an energy deficit in the formed islands. Otherwise, EVs will not be discharged. For this purpose, it is ensured that the available EVs energy will be used only if other DERs (DG units and ESSs) cannot fully supply the critical loads and implementation of emergency $\mathrm{V} 2 \mathrm{G}$ program will have the least possible convenience for $\mathrm{EV}$ users. Moreover, it is assumed that EVs in the parking lot can be charged during contingency events using excess energy of RESs;

3. Finally, total ENS of different MGs/sections is summed up to give the total value of $E N S_{m, h, s, j}$.
Moreover, note that the initial value of different ENS variables in the algorithm should be set to zero at the start of the algorithm. Furthermore, since it is assumed that no sectionalizing components are available in each MG/section [19], all components in a section/MG are in series from the viewpoint of reliability and all the contingencies with the same MTTR can be combined for reducing the number of contingency events that should be analyzed. In this case, the failure rate of the combined contingency event will be the sum of the associated failure rates.

Once the value of ENS index is estimated using the introduced algorithm, CIC can be calculated using Eq. (2). Estimation procedure for the OC will be discussed in the next section.

\section{Proposed model for estimating V2G operation costs}

4.1. Description of the battery wear cost model As mentioned earlier, concerns about EV battery wear due to V2G programs are considered to be a major obstacle to the implementation of such programs. In this context, some models have been proposed for estimation of the associated costs. In this paper, the model presented in the previous study [18] is used for this purpose. According to this model, battery wear price $(w p)$ multiplied by its total charged/discharged energy gives the total wear cost due to participation in V2G program. The value of $w p$ depends on driving pattern of the EV user and the amount of discharged energy of the $\mathrm{EV}$ in $\mathrm{V} 2 \mathrm{G}$ program run during normal operation of the grid.

\subsection{V2G annual operating cost model}

Total costs imposed on the EV user by participating in emergency $\mathrm{V} 2 \mathrm{G}$ programs can be divided into two terms; the first one is electricity cost associated with EV charging and the second one is the battery wear 
cost caused by battery discharge in V2G mode as well as the subsequent charge for bringing SOC to the initial level (before participation in $\mathrm{V} 2 \mathrm{G}$ program).

Accordingly, total cost incurred by the EV user because of participation $\left(\right.$ Cost $\left._{V 2 G}\right)$ can be calculated as follows:

$$
\operatorname{Cost}_{V 2 G}=2 w p E_{V 2 G}+\bar{\beta} E_{i n},
$$

where $E_{V 2 G}$ is the discharged energy of the battery during V2G program and $E_{i n}$ is the equivalent amount of energy received from the grid. Moreover, $\bar{\beta}$ is the average price of electricity in the associated charging process. Note that with participation in V2G program, the EV should undergo a discharge and the subsequent charge process so that its SOC level is brought to the initial level and the battery is degraded during both processes [18]. Therefore, a factor of 2 is considered for $E_{V 2 G}$.

On the other hand, the amount of energy delivered to the grid during emergency V2G program $\left(E_{\text {out }}\right)$ can be linked to the charged/discharged energy $\left(E_{V 2 G}\right)$ of the battery using charging and discharging efficiencies as follows:

$$
\begin{aligned}
& \eta^{\text {ch }} E_{i n}=E_{V 2 G}, \\
& E_{\text {out }}=\eta^{\mathrm{dch}} E_{V 2 G},
\end{aligned}
$$

in which $\eta^{\text {ch }}$ and $\eta^{\text {dch }}$ are charging and discharging efficiencies, respectively. Substituting Eqs. (6) and (7) in Eq. (5), Cost $_{V 2 G}$ can be expressed as follows:

$$
\text { Cost }_{V 2 G}=\left(\frac{2 w p}{\eta^{\mathrm{dch}}}+\frac{\bar{\beta}}{\eta^{\mathrm{dch}} \eta^{\mathrm{ch}}}\right) E_{\text {out }} .
$$

Eq. (8) gives total cost imposed on the EV user due to participation in $\mathrm{V} 2 \mathrm{G}$ program. Assuming that an additional $\varepsilon$ percent of Cost $_{V 2 G}$ should be paid as utilization cost of $\mathrm{V} 2 \mathrm{G}$ infrastructure and participation incentive to $\mathrm{EV}$ users, the total price of the $\mathrm{V} 2 \mathrm{G}$ program can be calculated as follows:

$$
c^{V 2 G}=\left(\frac{2 w p}{\eta^{\mathrm{dch}}}+\frac{\bar{\beta}}{\eta^{\mathrm{dch}} \eta^{\mathrm{ch}}}\right)\left(1+\frac{\varepsilon}{100}\right) .
$$

The calculated price gives the total cost that DSO should pay for $1 \mathrm{kWh}$ of the energy delivered to grid in emergency V2G program. Therefore, annual cost of implementing the introduced $\mathrm{V} 2 \mathrm{G}$ program can be calculated as follows:

$$
O C^{V 2 G}=c^{V 2 G}\left(E N S^{\text {Base }}-E N S^{V 2 G}\right),
$$

in which, $\mathrm{OC}^{V 2 G}$ is annual implementation cost of the program, and $E N S^{B a s e}$ and $E N S^{V 2 G}$ are ENS indices of the distribution system without and with implementation of the $\mathrm{V} 2 \mathrm{G}$ program, respectively. As a note, total annual energy extracted from the parking lot EVs during emergency events is equal to the difference between $E N S^{B a s e}$ and $E N S^{V 2 G}$. Thus, if multiplied by $c^{V 2 G}$, it gives annual operating cost.

\section{Reliability cost/worth assessment framework}

The overall structure of the proposed framework is illustrated in Figure 5. In this framework, all candidate emergency $\mathrm{V} 2 \mathrm{G}$ programs are identified in the first step. These programs may correspond to different locations of the parking lot, number of EVs participating in the program, and the adopted charging/discharging scheme. Subsequently, different cost terms associated with each program should be estimated and total annual costs calculated. The cost terms include the required investment costs for implementation of the candidate program, reduction in annual CIC due to the reliability improvements, and the associated annual operating costs. In this context, investment costs should be annualized based on the pertinent financial factors.

Once all the cost terms are aggregated, the program with the lowest cost can be selected as the optimal plan. Note that in this paper, it is assumed that parking lot location is fixed and it is intended to determine the optimal number of EVs participating in the emergency V2G program. Moreover, as discussed earlier, there will be no investment costs for the program in this paper. Besides, annual CIC can be estimated using the procedure delineated in Section 3 and annual operating cost of the program $\left(O C^{V 2 G}\right)$ is calculated using the method presented in Section 4 .

\section{Case studies}

\subsection{Test system and main assumptions}

For illustrating applicability of the proposed framework, emergency V2G programs of EV parking lot

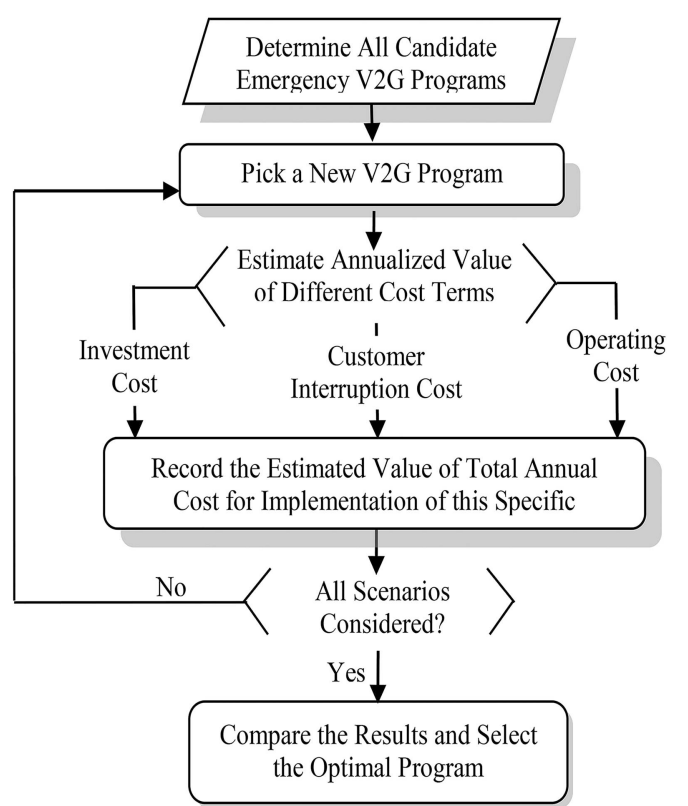

Figure 5. Proposed framework for selecting the optimal emergency $\mathrm{V} 2 \mathrm{G}$ program. 
will be deployed in the IEEE 34-node test system for the reliability improvement purpose [29]. This feeder has been modified by integration of three MGs and an EV parking lotm as shown in Figure 6. Moreover, it is assumed that MGs and distribution feeder can exchange power via PCCs and interconnections between MGs [19]. Power transfer capacities of each interconnection and PCC are 0.3 MW and 1.2 MW, respectively.

The MGs include Micro-Turbines (MTs), Wind Turbine (WT) generators, Photovoltaic (PV) units, Energy Storage Systems (ESSs), and loads. DER characteristics and reliability data of MMG system components are set according to the previous study [19]. It is assumed that feeder and three MGs supply residential loads and have the same hourly profile [19]. Moreover, the value of $1.2 \mathrm{US} \$ / \mathrm{kWh}$ is used in the estimation of CIC in this paper [30]. In addition, as mentioned earlier, the decentralized operating schemes introduced previously [19] are used in the simulation of the MMG systems in the case studies. Annual peak loads of each MG and feeder are $1 \mathrm{MW}$ and $0.4 \mathrm{MW}$, respectively. Moreover, no sectionalizing devices are considered within the feeder or MGs.

\subsection{Results and analysis}

As the basic case, it is assumed that $200 \mathrm{EVs}$ will participate in the emergency V2G program. As mentioned earlier, 4 different strategies are considered for charge scheduling of EVs during normal operation of the grid. The results obtained for ENS index in different cases are summarized in Table 3. It should be noted that Average Energy Not Supplied (AENS) [22] index is equal to ENS divided by the number of customers. The number of customers in the distribution system under study is 1300 .

Comparing the results, it can be seen that charging strategy during normal operation significantly affects reliability of the system. According to the results, dumb charging causes the highest reliability improvement. It is because in this case, the batteries are charged with maximum allowable power and therefore, their SOC levels will rise more rapidly. As a result, their daily average available energy will be higher than that in the other charging strategies. This argument can be confirmed by looking at Figure 3. On the other hand, when $\mathrm{V} 2 \mathrm{G}$ programs are implemented during normal operation, the reliability improvement level will have the lowest value, since EVs cannot maintain a high daily average SOC level due to discharging in times of high electricity price [7].

Sensitivity of AENS index to the number of participating EVs is summarized in Table 4. Note that in this case, it is assumed that EVs also participate in V2G programs during normal operation. i.e., charging case of "V2G" is investigated. As can be observed, reliability improves as the number of participating $\mathrm{EVs}$ is increased. However, reliability improvement level gradually decreases at higher EV numbers. This observation can be justified as follows. Discharged energy of parking lot can reduce the load curtailments in the case of outages in the external grid. Therefore, it will have a limited impact on reliability improvement of the MMG system.

The result of cost/worth analysis is reflected in Figure 7 . The values of $w p, \bar{\beta}$, and $\varepsilon$ are respectively set to $0.0868 \mathrm{US} \$ / \mathrm{kWh}, 0.0917 \mathrm{US} \$ / \mathrm{kWh}$, and $10 \%$ for this study. Moreover, charging and discharging efficiencies are both set to $93 \%$.

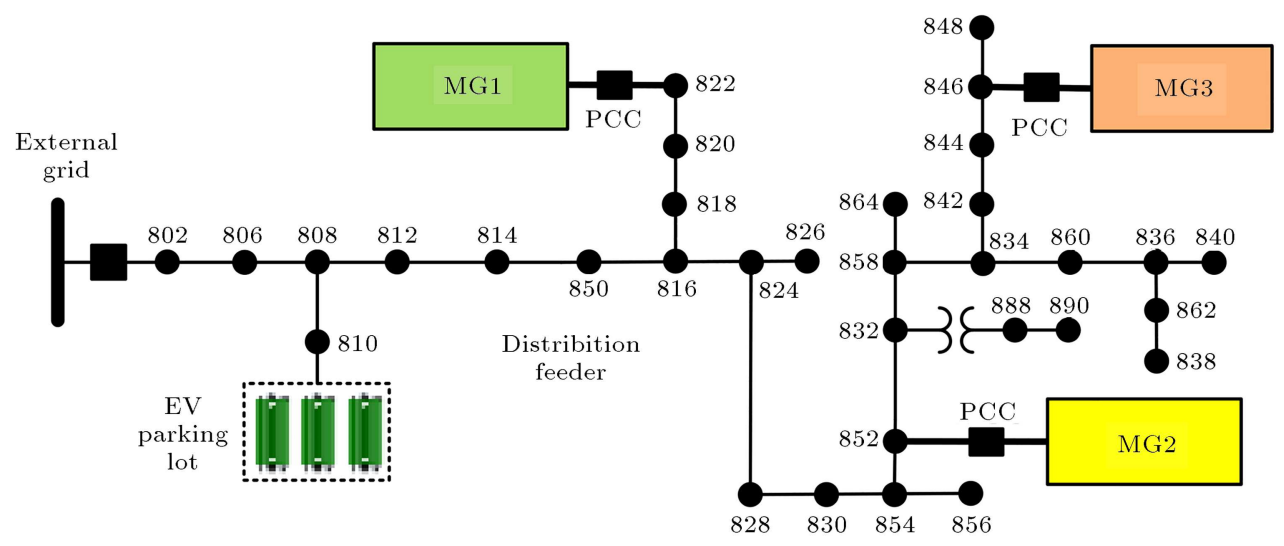

Figure 6. Single-line diagram of the MMG system under study.

Table 3. AENSs for different charge scheduling strategies in the basic case.

\begin{tabular}{ccccc}
\hline Charging strategy & No V2G & V2G & Uniform charging & Dumb charging \\
\hline AENS (kWh/cust.Yr) & 13.165 & 14.029 & 12.920 & 12.858 \\
\hline
\end{tabular}


Table 4. Reliability improvement versus the number of participating EVs.

\begin{tabular}{cccccc} 
Number of EVs & 0 & 100 & 200 & 300 & 400 \\
AENS (kWh/cust.Yr) & 14.510 & 14.252 & 14.029 & 13.802 & 13.564 \\
\hline
\end{tabular}

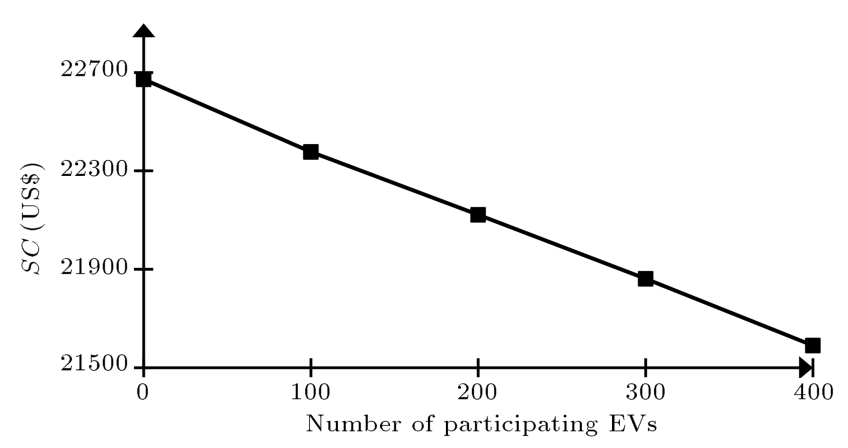

Figure 7. Annual social cost versus number of participating EVs.

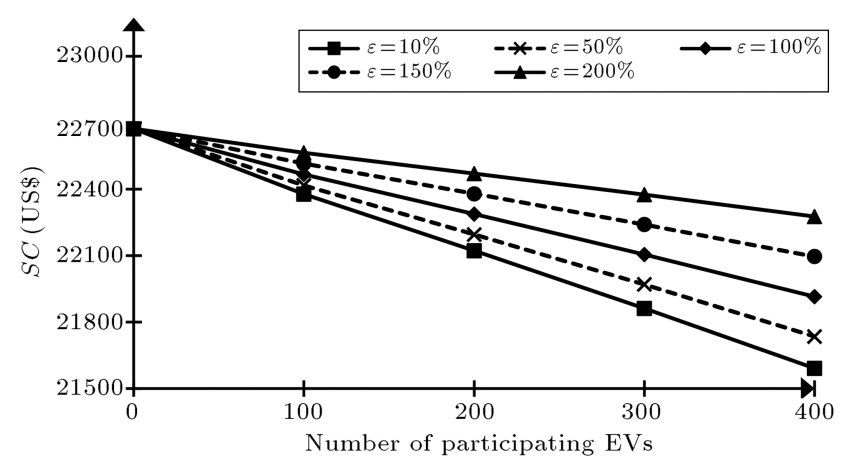

Figure 8. Annual social cost versus number of participating EVs for different values of $\varepsilon$.

As can be seen, annual social cost of the system decreases with increase in the number of participating EVs. In other words, implementation of emergency $\mathrm{V} 2 \mathrm{G}$ programs is cost-effective under the assumed circumstances. This conclusion is not surprising, since the value obtained for $c^{V 2 G}$ in this study is $0.3219 \mathrm{US} \$ / \mathrm{kWh}$. Although this value is almost three times higher than average electricity price, it is much lower than the typical values of VOLL and this fact justifies the application of the assumed program.

\subsection{Sensitivity analysis}

In this section, a sensitivity analysis is conducted for the value of $\varepsilon$ and the results are shown in Figure 8 . As can be observed, total annual social cost increases as the value of $\varepsilon$ is increased. However, $\mathrm{SC}$ graph is monotonically decreasing for all values of $\varepsilon$. This is because in all cases, price of $\mathrm{V} 2 \mathrm{G}$ programs $\left(c^{V 2 G}\right)$ is still lower than VOLL. Therefore, total SC will decrease as participation of $\mathrm{EV}$ increases. In other words, implementation of emergency V2G programs will be cost-effective under these conditions as far as financial costs are concerned. It should be noted, however, that in order to gain a more comprehensive assessment of the associated costs and benefits, the impacts of such programs on the convenience of EV users should also be modeled and evaluated [31].

\section{Conclusions}

This paper presented a framework for optimal design of EV parking lot emergency V2G programs for reliability improvement of active distribution system. This framework determined the optimal number of participating EVs based on cost/worth analysis. In this context, impacts of such programs on different system costs as well the obtained worth were discussed. Moreover, appropriate models were introduced for the calculations. The presented framework was implemented on a test system and performance of $\mathrm{V} 2 \mathrm{G}$ programs under different conditions were investigated. The obtained results suggested that under the assumed circumstances, implementation of emergency V2G programs was costeffective as far as financial costs were concerned.

\section{Acknowledgement}

This work is supported by Iran's "National Elites Foundation."

\section{References}

1. Muratori, M. "Impact of uncoordinated plug-in electric vehicle charging on residential power demand", Nature Energy, 3(3), pp. 193-201 (2018).

2. DeForest, N., MacDonald, J.S., and Black, D.R. "Day ahead optimization of an electric vehicle fleet providing ancillary services in the Los Angeles air force base vehicle-to-grid demonstration", App. Energy, 210, pp. 987-1001 (2018).

3. Shams, H., Sadeghfam, A., Rostami, N., et al. "Exact approach for charging of pevs with $\mathrm{V} 2 \mathrm{G}$ capability to improve micro-grid reliability", IET Gen., Trans. \& Dist., 13(16), pp. 3690-3695 (2019).

4. Faddel, S., Aldeek, A., Al-Awami, A.T., et al. "Ancillary services bidding for uncertain bidirectional V2G using fuzzy linear programming", Energy, 160, pp. 986-995 (2018).

5. Chandra Mouli, G., Kefayati, M., Baldick, R., et al. "Integrated PV charging of EV fleet based on energy prices, V2G and offer of reserves", IEEE Trans. on Smart Grid, 10(2), pp. 1313-1325 (2018).

6. Arias, N.B., Hashemi, S., Andersen, P.B., et al. "V2G enabled EVs providing frequency containment reserves: field results", IEEE Int. Conf. on Ind. Tech. (ICIT) (2018). 
7. Farzin, H., Fotuhi-Firuzabad, M., and Moeini, M. "Reliability studies of modern distribution systems integrated with renewable generation and parking lots", IEEE Trans. on Sust. Energy, 8(1), pp. 431-440 (2017).

8. Domínguez-Navarro, J., Dufo-López, R., Yusta-Loyo, J., et al. "Design of an electric vehicle fast-charging station with integration of renewable energy and storage systems", Int. J. of Elec. Power \& Energy Sys., 105, pp. 46-58 (2019).

9. Dong, X., Mu, Y., Xu, X., et al. "A charging pricing strategy of electric vehicle fast charging stations for the voltage control of electricity distribution networks", App. Energy, 225, pp. 857-868 (2018).

10. Bryden, T.S., Hilton, G., Cruden, A., et al. "Electric vehicle fast charging station usage and power requirements", Energy, 152, pp. 322-332 (2018).

11. Lin, C., Yao, C., Jin, L., et al. "Power system reliability assessment with electric vehicle integration using battery exchange mode", IEEE Trans. on Sust. Energy, 4(4), pp. 1034-1042 (2013).

12. Farzin, H., Fotuhi-Firuzabad, M., and Moeini-Aghtaie, M. "Reliability studies of modern distribution systems integrated with renewable generation and parking lots", IEEE Trans. Sust. Energy, 8(1), pp. 431-440 (2017).

13. Liu, Z., Wang, D., Jia, H., et al. "Aggregation and bidirectional charging power control of plug-in hybrid electric vehicles: Generation system adequacy analysis", IEEE Trans. on Sust. Energy, 6(2), pp. 325-335 (2015).

14. Xu, N. and Chung, C. "Well-being analysis of generating systems considering electric vehicle charging", IEEE Trans. on Power Syst, 29(5), pp. 2311-2320 (2014).

15. Xu, N.Z. and Chung, C.Y. "Reliability evaluation of distribution systems including vehicle-to-home and vehicle-to-grid", IEEE Trans. on Power Syst., 31(1), pp. 759-768 (2016).

16. Moeini, M., Farzin, H., Fotuhi-Firuzabad, M., et al. "Generalized analytical approach to assess reliability of renewable-based energy hubs", IEEE Trans. on Power Syst., 32(1), pp. 368-377 (2017).

17. Ahmadian, A., Sedghi, M., Elkamel, A., et al. "Plugin electric vehicle batteries degradation modeling for smart grid studies: review, assessment and conceptual framework", Ren. Sust. Energy Rev., 81, pp. 2609-2624 (2018).

18. Farzin, H., Fotuhi-Firuzabad, M., and Moeini, M. "A practical scheme to involve degradation cost of lithiumion batteries in vehicle-to-grid applications", IEEE Trans. on Sust. Energy, 7(4), pp. 1730-1738 (2016).

19. Farzin, H., Fotuhi-Firuzabad, M., and Moeini-Aghtaie, M. "Role of outage management strategy in reliability performance of multi-microgrid distribution systems", IEEE Trans. on Power Syst., 33(3), pp. 2359-2369 (2018).
20. Farzin, H., Moeini-Aghtaie, M., and Fotuhi-Firuzabad, M. "A hierarchical scheme for outage management in multi-microgrids", Int. Trans. on Elec. Energy Syst., 26(9), pp. 2023-2037 (2016).

21. Farzin, H., Ghorani, R., Fotuhi-Firuzabad, M., et al. "A market mechanism to quantify emergency energy transactions value in a multi-microgrid system", IEEE Trans. on Sust. Energy, 10(1), pp. 426-437 (2019).

22. Billinton, R. and Allan, R.N., Reliability Evaluation of Power Systems, Plenum press, New York, USA (1984).

23. Li, W., Reliability Assessment of Electrical Power Systems Using Monte Carlo Methods, Springer Science \& Business Media, Germany (1994).

24. Farzin, H., Monadi, M., and Rodriguez, P. "A cost/worth analysis framework for reliability enhancement of multi-microgrid distribution systems", Presented at IEEE PES General Meeting (2019).

25. 2009 National Household Travel Survey, U.S. Department of Transportation, Federal Highway Administration (2009).

26. Farzin, H., Moeini-Aghtaie, M., and Fotuhi-Firuzabad, M. "Reliability studies of distribution systems integrated with electric vehicles under battery-exchange mode", IEEE Trans. on Power Del., 31(6), pp. 24732482 (2016).

27. Shafiee, S., Fotuhi-Firuzabad, M., and Rastegar, M. "Investigating the impacts of plug-in hybrid electric vehicles on power distribution systems", IEEE Trans. on Smart Grid, 4(3), pp. 1351-1360 (2013).

28. Conti, S., Rizzo, S.A., El-Saadany, E.F., et al. "Reliability assessment of distribution systems considering telecontrolled switches and microgrids", IEEE Trans. on Power Syst, 29(2), pp. 598-607 (2014).

29. http://www.ewh.iee.org/soc/pes/dsacom/testfeeders/ index.html, Accessed Aug. (2019).

30. Simab, M. and Haghifam, M.R. "Quality performance based regulation through designing reward and penalty scheme for electric distribution companies", Int. J. of Elec. Power \& Energy Syst., 43(1), pp. 539-545 (2012).

31. Magome, N. and Tamura, S. "A new decentralized control of EVs for load frequency control retaining EV users' convenience", IEEE Int. Tele. Energy Conf. (INTELEC) (2018).

\section{Biographies}

Hossein Farzin received the BSc and $\mathrm{PhD}$ degrees in Electrical Engineering from Sharif University of Technology, Tehran, Iran, in 2011 and 2016, respectively. He is currently an Assistant Professor in the Electrical Engineering Department, Shahid Chamran University of Ahvaz, Ahvaz, Iran. His research interests include microgrids design and optimization, integration of distributed energy resources and electric vehicles in smart grid, and power system reliability and resilience. 
Mehdi Monadi received his BSc degree from Shahid Chamran University of Ahvaz, Ahvaz, Iran, and his MSc degree from Shahrood University of Technology, Iran. Then, he received his $\mathrm{PhD}$ degree in Electrical Engineering from the Technical University of Catalonia (UPC), Barcelona, Spain, in 2016. In 2015, he was a visiting $\mathrm{PhD}$ student in the $\mathrm{KTH}$ Royal Institute of Technology, Stockholm, Sweden. Since 2018, he has been with Shahid Chamran University of Ahvaz, Ahvaz, Iran, as an Assistant Professor. He has also been with South Steel Pishgaman Company (SSPC) as a consultant in electrical and automation projects of Khuzestan Steel Company (KSC) since 2018. His research interests include protection of active/smart distribution systems, and dc distribution and transmission systems. 